\title{
APPLICATION OF AN ESTIMATOR-FREE INFORMATION CRITERION (WIC) TO APERTURE SYNTHESIS IMAGING
}

\section{ISHIGURO}

The Institute of Statistical Mathematics, 4-6-7 Minami Azabu, Minatoku, Tokyo, 106 Japan

\section{K.I. MORITA}

Nobeyama Radio Observatory, National Astronomical observatory, Nobeyama, MinamiSaku Nagano, 384-13

\section{ISHIGURO}

Nobeyama Radio Observatory, National Astronomical observatory, Nobeyama, Minami Maki-mura, Minami Saku-gun, Nagano-ken, 384-13 Japan

ABSTRACT A statistical critericn for stopping CLEAN procedure is proposed. The criterion is called $W I C$ and an estimator of KullbackLeibler information quantity which is a measure of the goodness of statistical models. A numerical example is given.

\section{INTRODUCTION}

Visibility Data

Visibility $z_{j}$ observed at time $j$ can be expressed in terms of "true visibility" defined by the brightness distribution $T(x, y)$ and observation noise $\varepsilon_{j}$ :

$$
\begin{gathered}
z_{j}=\tilde{T}\left(u_{j}, v_{j}\right)+\varepsilon_{j} \quad(j=1,2, \ldots, N) \\
\tilde{T}(u, v)=\iint \exp \{2 \pi i(x u+y v)\} T(x, y) d x d y,
\end{gathered}
$$

where $\left\{\left(u_{j}, v_{j}\right)\right\}$ is a set of points in the $u$-v plane. If $\varepsilon_{j}$ can be regarded as a random variable, then the vector $z=\left(z_{1}, z_{2}, \ldots, z_{N}\right)$ is an $N$ dimensional complex random variable. Let $f(z)$ denote the probability density function $(P D F)$ of $z$.

\section{Statistical Modeling}

Assume that a model of $\mathrm{T}(x, y)$ is given by 


$$
T_{M}(x, y)=\sum_{m=1}^{M} w_{m} K\left(x-x_{m}, y-y_{m}\right)
$$

where $K(x, y)$ is a suitably fixed kernel function.

Substituting this equation for $T(x, y)$ in Eq. (1), and assuming $\left\{\varepsilon_{j}\right\}$ be a mutually independent complex normal random numbers with mean 0 and variance $\sigma^{2}$, a statistical model of data $z$ is defined which is parametrized by $\left(w_{1}, x_{1}, y_{1}\right),\left(w_{2}, x_{2}, y_{2}\right), \ldots,\left(w_{M}, x_{M}, y_{M}\right)$ and $\sigma^{2}$. Let us denote this model by $f\left(z \mid\left(w_{1}, x_{1}, y_{1}\right),\left(w_{2}, x_{2}, y_{2}\right), \ldots,\left(w_{M}, x_{M}, y_{M}\right), \sigma_{2}\right)$.

\section{Image formation}

When an estimate $\left\{\left(\hat{w}_{1}, \hat{x}_{1}, \hat{y}_{1}\right),\left(\hat{w}_{2}, \hat{x}_{2}, \hat{y}_{2}\right), \ldots,\left(\hat{w}_{M}, \hat{x}_{M}, \hat{y}_{M}\right)\right\}$ is given, it is natural to estimate $\sigma^{2}$ by

$$
\dot{\sigma}^{2}=\frac{1}{2 N} \sum_{j=1}^{N}\left|z_{j}-\tilde{T}_{M}\left(u_{j}, v_{j}\right)\right|^{2}
$$

where $\tilde{T}_{M}(u, v)$ is defined by substituting estimate $T_{M}(x, y)$ in Eq.(2). The model of data $z, f\left(z \mid\left(\hat{w}_{1}, \hat{x}_{1}, \hat{y}_{1}\right),\left(\hat{w}_{2}, \hat{x}_{2}, \hat{y}_{2}\right), \ldots,\left(\hat{w}_{M}, \hat{x}_{M}, \hat{y}_{M}\right), \hat{\sigma}^{2}\right)$ should be an estimate of the true $P D F f(z)$.

\section{INFORMATION CRITERION}

The goodness of statistical models is measured by the expected log likelihood defined by

$$
E L L=\int f(z) \log f\left(z \mid\left(\hat{w}_{1}, \hat{x}_{1}, \hat{y}_{1}\right),\left(\dot{w}_{2}, \dot{x}_{2}, \hat{y}_{2}\right), \ldots,\left(\dot{w}_{M}, \hat{x}_{M}, \dot{y}_{M}\right), \hat{\sigma}^{2}\right) d z
$$

A larger value of $E L L$ means a better fit of a model.

Ishiguro \& Ishiguro (1980) successfully applied the $E L L$ minimization procedure to the aperture synthesis data analysis. They employed the maximum likelihood method to the model fitting, and used $A I C$ ( Akaike, $1973)$ as the estimator of $E L L$. The example was the one-dimensional imaging problem. The extension of the proposed method, however, to the twodimensional imaging was difficult. Two dimensionlal models are too complex to be fitied by the maximum likelihood method and it is a vital condition for the use of $A I C$ that the parameter estimation is done by the maximum likelihood method. 
WIC

We propose the use of WIC, an estimator-free information criterion, when the model fitting is not done by the maximum likelihood method. $W I C$ is an Extension of $A I C$ which is defined by

$$
\begin{aligned}
& W I C=-2 \times \log f\left(z \mid\left(\hat{w}_{1}, \hat{x}_{1}, \hat{y}_{1}\right),\left(\hat{w}_{2}, \dot{x}_{2}, \hat{y}_{2}\right), \ldots,\left(\hat{w}_{M}, \dot{x}_{M}, \hat{y}_{M}\right), \dot{\sigma}^{2}\right) \\
& +2 \times E_{2} \cdot\left\{\log f\left(z^{*} \mid\left(\hat{w}_{1}^{*}, \hat{x}_{1}^{*}, \hat{y}_{1}\right),\left(\hat{w}_{2}^{*}, \dot{x}_{2}^{*}, \hat{y}_{2}^{*}\right), \ldots,\left(\hat{w}_{M}^{*}, \hat{x}_{M}^{*}, \hat{y}_{M}^{*}\right), \hat{\sigma}^{-2}\right)\right. \\
& \left.-\log f\left(z \mid\left(\hat{w}_{1}^{*}, \dot{x}_{1}^{*}, \hat{y}_{1}^{*}\right),\left(\bar{w}_{2}^{*}, \bar{x}_{2}^{*}, \dot{y}_{2}\right), \ldots,\left(\bar{w}_{M}^{*}, \dot{x}_{M}^{*}, \dot{y}_{M I}^{*}\right), \hat{\sigma}^{* 2}\right)\right\}
\end{aligned}
$$

where $z^{\bullet}$ is a simulated data obtained by the resampling technique $\left\{\left(\hat{w}_{1}^{*}, \hat{x}_{1}^{*}, \hat{y}_{1}^{*}\right),\left(\hat{w}_{2}^{*}, \hat{x}_{3}^{*}, \hat{y}_{2}^{*}\right), \ldots,\left(\hat{w}_{M}^{*}, \hat{x}_{M}^{*}, \hat{y}_{M}^{*}\right)\right\}$ and $\hat{\sigma}^{-2}$ are estimates of parameters based on the data $z^{*}, E_{2}$. denotes the expectation with respect to the variation of $z *$, which is practically computed by the Monte Carlo method.

\section{A NUMERICAL EXAMPLE}

We applied the WIC minization procedure for the choice of the number of $C L E A N$ component.

A set of data was produced, asuming the structure shown in Fig. Ia. The $C L E A N$ process proceeds as shown in Figs.1d through if. The $W I C$ values indicate that we should stop around $M=600$. ELL values which could be computed only when the data is artificially generated supports this choice. Visual inspection also endoses this choice.
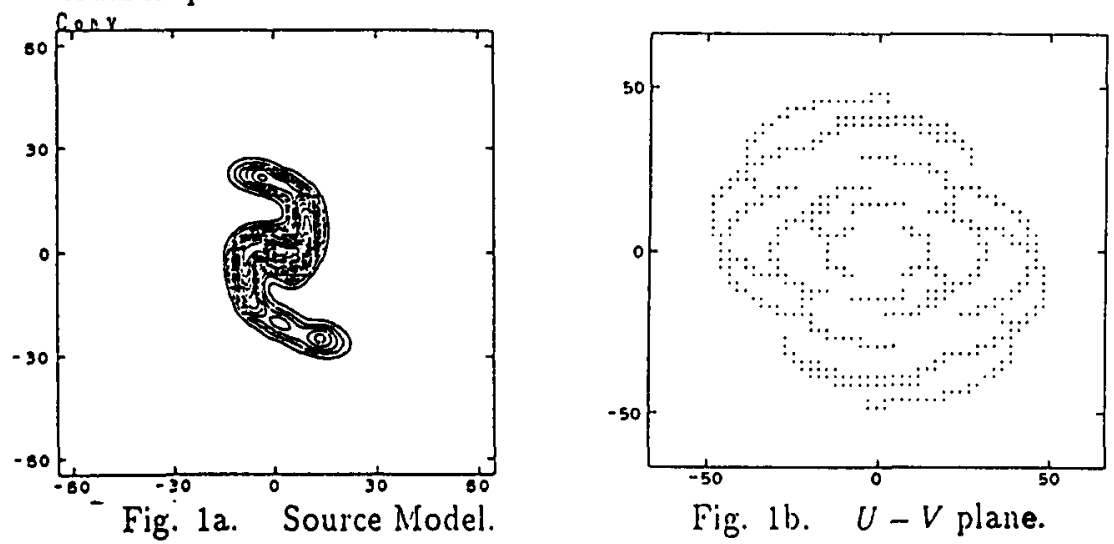

Fig. 1b. $U-V$ plane. 


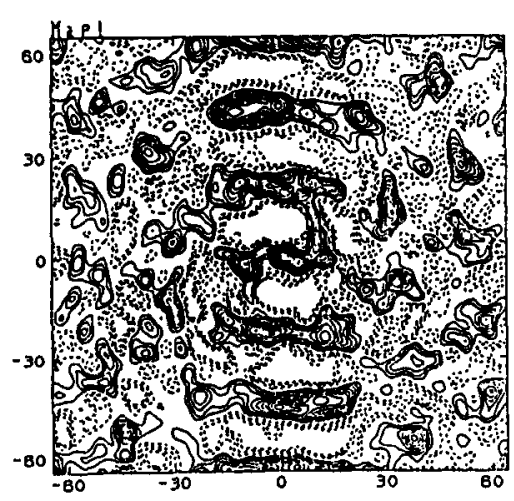

Fig. 1c. Synthesized Map.

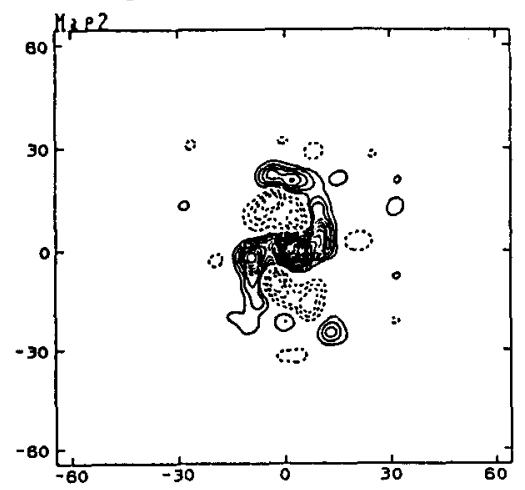

Fig. 1e. Clean Map $(\mathrm{N}=600)$.

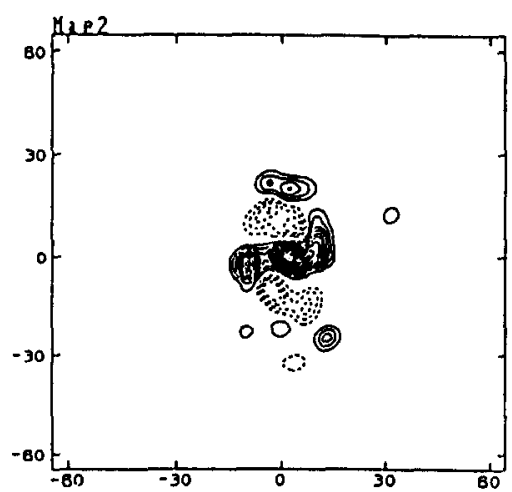

Fig. 1d. Clean Map $(M=200)$.

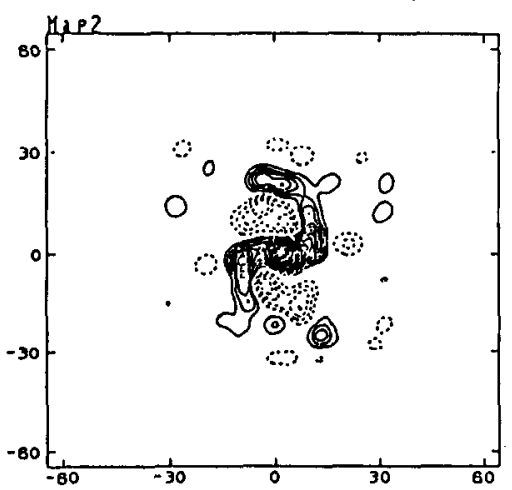

Fig. 1f. Clean Map (M=1000).

TABLE II Analysis of complex data

\begin{tabular}{rll}
\hline$M$ & $W I C$ & $-2 E L L$ \\
\hline 100 & 296.17 .9 & 29643.5 \\
200 & 29424.2 & 29411.0 \\
300 & 29334.6 & 29312.8 \\
400 & 29298.2 & 29269.0 \\
500 & 29284.2 & 29248.1 \\
600 & 29230.6 & 29233.2 \\
700 & 29281.6 & 29235.0 \\
500 & 29284.3 & 29233.3 \\
900 & 29287.5 & 29235.2 \\
1000 & 29291.1 & 29237.2 \\
\hline
\end{tabular}




\section{ACKNOWLEDGEMENTS}

This work is partly supported by the Grant-in-Aid for Scientific Research provided by The Ministry of Education, Science and Culture, and carried out under the ISM Cooperative Research Program(90-ISM.CRP-45) of the Institute of Statistical Mathematics.

\section{REFERENCES}

Akaike, H. 1973, in Proc. 2nd Inter. Symp. on Information Theory ed

B. N. Petyov and F. Csai, A kademiai Kiado, Budapest, pp.267-281.

Efron, B. 1979, The Annals of Statistics, 7, 1, pp.1-26.

Sakamoto, Y., Ishiguro, M. \& Kitagawa, G. 1986, Akaike Information Criterion Statistics, D.Reidel Publishing Company, Dordrecht/Tokyo.

Schwartz, U. J. 1977, Main Journal.

Wong, W. H. 1983, JASA, 78, 382, pp.461-463. 


\section{APPENDIX: A SHORT DICTIONARY OF STATISTICAL TERMS}

AIC: An estimate of Information Criterion proposed by Akaike. It is defined by

$$
\left.A I C=-2 \log \max _{\theta} f(x \mid \theta)+2 \text { number of free parameters }\right\},
$$

where $f$ is a statistical model of data $x$ with parameter $\theta$. It is a measure of the badness of the statistical models.

Expected log likelihood: Let $f(x)$ and $f(x \mid \theta)$ denotes the true $P D F$ of data $x$ and its model, respectively. Then the expected log likelihood is defined by

$$
\int f(x) \log f(x \mid \theta) d x .
$$

The larger value of the expected log likelihood means a better fit of the model to the real $P D F$.

Log likelihood: Let $f(x \mid \theta)$ be a statistical model. If $x$ is fixed at the value of given data, $f$ can be regarded as the function of parameter $\theta$ only. This function is called likelihood function of $\theta$. Log likelihood function is the logarithm of likelihood function.

MLE: Maximum likelihood estimate. The estimated parameter of a statistical model obtained by maximizing the $(\log )$ likelihood function.

Resampling: When we have a set of data, and need to generate similar set of data, there are two possible way. One is to fit a $P D F$ model to the data and generate simulation data using this fitted $P D F$. The other way is the "resampling". Suppose that we have the set of data

$$
\left\{d_{1}, d_{2}, \ldots d_{N}\right\}
$$

then the resampled data is obtained by randomly chosing $N$ values from this set of data replacing the chosen value every time. Formal expression of the resampled data could be

$$
\left\{d_{j(1)}, d_{j(2)}, \ldots d_{j(N)}\right\}
$$

where $\{j(1), j(2), \ldots, j(N)\}$ is a sequence of independent random numbers uniformly distributed on the interval $[1, N]$.

Statistical Model: Usually a parametrized family of probability (density) function. 